\title{
THE NEW SUB-EQUATION METHOD FOR GENERAL KAUP-KUPERSHMIDT EQUATION FROM CAPILLARY GRAVITY WAVES WITH CONFORMABLE DERIVATIVE
}

\author{
HADI REZAZADEH ${ }^{1}$, ALI KURT ${ }^{2, *}$, MOSTAFA ESLAMI ${ }^{3}$, MOHAMMAD MIRZAZADEH $^{4}$, ORKUN \\ TASBOZAN $^{5}$, OZAN ÖZKAN ${ }^{6}$
}

\author{
${ }^{1}$ 1Faculty of Engineering Technology, Amol University of Special Modern Technologies, Amol, Iran \\ ${ }^{2}$ Department of Mathematics, Faculty of Science and Art, Pamukkale University, Denizli, Turkey \\ ${ }^{3}$ Department of Mathematics, Faculty of Mathematical Sciences, University of Mazandaran, Babolsar, Iran \\ ${ }^{4}$ Department of Engineering Sciences, Faculty of Technology and Engineering, East of Guilan, University of Guilan, Rudsar-Vajargah, Iran \\ ${ }^{5}$ Department of Mathematics, Faculty of Science and Art, Mustafa Kemal University, Hatay, Turkey \\ ${ }^{6}$ Department of Mathematics, Faculty of Science, Konya Selçuk University, Hatay, Turkey \\ *Corresponding Author: pau.dr.alikurt@gmail.com
}

Received Feb. 14, 2019

\begin{abstract}
Aвstract. In this present paper, authors obtained the exact solution of time fractional general Kaup-Kupershmidt equation where the fracitonal derivative operator is in conformable sense by using the new sub-equation method. This method implemented firstly in the literature to a fractional partial differential equation.
\end{abstract}

2010 Mathematics Subject Classification. 35R11; 35A20; 35C05.

Key words and phrases. conformable fractional derivative; the new sub-equation method; fractional general KaupKupershmidt; exact solutions.

\section{INTRODUCTION}

The generalizations of differentiation and integration with integer orders are called fractional calculus. In the last decades, interest to fractional calculus has been increasing considerably because of its huge application area in various fields such as physics, engineering, dynamical systems, control systems [1-4]. As a result of this interest, many powerful methods to solve fractional differential equations were presented by many authors. For example Kurt et. al. [5] obtained approximate anaytical solutions of Whitham-Broer-Kaup Equation by using homotopy analysis with Caputo derivative.Çelik et. al. [6] used Crank-Nicolson method for solvingfractional diffusion equation with the Riesz fractional derivative. Tasbozan et. al. [7] employed finite element method for obtaining the approximate solutions of diffusion equation with Riemann-Liouville fractional derivative. As it is seen from the the references given above, authors applied numerical methods to obtain the approximate solutionsof considered equations. Because scientists can not obtain the anaytical solutions for Caputo, Riemann-Liouville and the Riesz fractional derivatives. But newly defined conformable fractional derivative let us to obtain the

DOI: 10.28924/APJM/6-3

(C)2019 Asia Pacific Journal of Mathematics 
analytical solutions of the considered equations by using wave transform [8] and chain rule [9]. For instance Korkmaz et. al. [10] used two different methods to obtain the analytical solution of time-fractional parabolic equation with exponential nonlinearity. Çenesiz et. al. [11] employed first integral method to get the exact solutions of conformable fractional Burgers' type equations. Kurt et. al. [12] considered method to get the analytical solutions of conformable fractional Nizhnik-Novikov-Veselov system. For further details see papers [13-22]

In this study we regard the conformable time-fractional general Kaup-Kupershmidt equation ( For $\alpha=1$ see [23])

$$
\frac{\partial^{\alpha} u}{\partial t^{\alpha}}+\frac{1}{5} \gamma^{2} u^{2} \frac{\partial u}{\partial x}+\frac{5}{2} \gamma \frac{\partial u}{\partial x} \frac{\partial^{2} u}{\partial x^{2}}+\gamma u \frac{\partial^{3} u}{\partial x^{3}}+\frac{\partial^{5} u}{\partial x^{5}}=0
$$

where $\alpha \in(0,1]$. Also, in the case $\gamma=3$, Eq. (1.1) reduces to the standard Kaup-Kupershmidt equation

$$
\frac{\partial^{\alpha} u}{\partial t^{\alpha}}+20 u^{2} \frac{\partial u}{\partial x}+25 \frac{\partial u}{\partial x} \frac{\partial^{2} u}{\partial x^{2}}+10 u \frac{\partial^{3} u}{\partial x^{3}}+\frac{\partial^{5} u}{\partial x^{5}}=0
$$

The fifth order Kaup-Kupershmidt equation (1.1) is one of the solitonic equations related to the integrable cases of the Henon-Heiles system and belongs to the completely integrable hierarchy of higher order KdV equations [24]. These Eq (1.1) and Eq (1.2) for $\alpha=1$ studied using various techniques, for example the extended tanh method [23], the Fan sub-equation method [24], the Projective Riccati equation method [25], the simplified Hirota's method [26].

The rest of the work is arranged as follows: In Section 2, the definition of conformable fractional derivative is introduced, and the basic properties of fractional derivative are investigated. In Section 3, we give a description of the the new sub-equation method. In Section 4, we employ the new sub-equation method to conformable time-fractional general Kaup-Kupershmidt equation. Finally, we give a concluding remarks in section 5.

\section{Conformable Fractional Calculus}

Conformable fractional calculus firstly mentioned by R. Khalil et. al. [27] is well behaved, applicable and obeys many rules that known derivative and integral satisfies.

Definition 2.1. Let $f:[0, \infty) \rightarrow \mathbb{R}$ be a function. $\alpha^{\text {th }}$ order "conformable fractional derivative" of $f$ is defined by

$$
T_{\alpha}(f)(t)=\lim _{\varepsilon \rightarrow 0} \frac{f\left(t+\varepsilon t^{1-\alpha}\right)-f(t)}{\varepsilon}
$$

for all $t>0, \alpha \in(0,1)$. If $f$ is $\alpha$ differentiable in some $(0, a), a>0$ and

$$
\lim _{t \rightarrow 0} f(t)
$$

exists, then define

$$
f^{(\alpha)}(0)=\lim _{t \rightarrow 0^{+}} f^{(\alpha)}(t) .
$$

But other fractional derivatives such as Caputo, Riemann-Liouville, Grünwald so not satisfy basic rules. For instance

(1) Let $\lambda$ be a constant and $\alpha \in R$. So $D_{a}^{\alpha}(\lambda) \neq 0$ for Riemann-Liouville derivative. 
(2) All fractional derivatives do not satisfy the known formula of the derivative of the product of two functions.

$$
D_{a}^{\alpha}(f g) \neq f D_{a}^{\alpha}(g)+g D_{a}^{\alpha}(f)
$$

(3) All fractional derivatives do not satisfy the known formula of the derivative of the quotient of two functions.

$$
D_{a}^{\alpha}\left(\frac{f}{g}\right) \neq \frac{g D_{a}^{\alpha}(f)-f D_{a}^{\alpha}(g)}{g^{2}}
$$

(4) All fractional derivatives do not satisfy the chain rule.

$$
D_{a}^{\alpha}(f \circ g) \neq f^{(\alpha)}(g(t)) g^{(\alpha)}(t)
$$

(5) All fractional derivatives do not satisfy

$$
D^{\alpha} D^{\beta} f=D^{\alpha+\beta} f
$$

in general.

(6) The Caputo derivative assumes that the function $f$ is differentiable.

This new definition satisfies the properties which are given in the following theorem.

Theorem 2.1. Let $\alpha \in(0,1)$ and $f, g$ be $\alpha$-differentiable at point $t>0$. Then

(1) $T_{\alpha}(c f+d g)=c T_{\alpha}(f)+d T_{\alpha}(g)$,for all $a, b \in \mathbb{R}$.

(2) $T_{\alpha}\left(t^{p}\right)=p t^{p-\alpha}$ for allp $\in \mathbb{R}$.

(3) $T_{\alpha}(\lambda)=0$ for all constant functions $f(t)=\lambda$.

(4) $T_{\alpha}(f g)=f T_{\alpha}(g)+g T_{\alpha}(f)$.

(5) $T_{\alpha}\left(\frac{f}{g}\right)=\frac{g T_{\alpha}(g)-f T_{\alpha}(f)}{g^{2}}$

(6) If, in addition to $f$ is differentiable, then $T_{\alpha}(f)(t)=t^{1-\alpha} \frac{d f}{d t}$.

\section{Description of THE NeW SUb-eQuation Method}

A general fractional nonlinear wave equation can be written as

$$
F\left(u, \frac{\partial^{\alpha} u}{\partial t^{\alpha}}, \frac{\partial u}{\partial x}, u \frac{\partial u}{\partial x}, u^{2} \frac{\partial u}{\partial x}, \frac{\partial^{2} u}{\partial x^{2}}, \ldots\right)=0 .
$$

We seek its travelling wave solution $u(\xi)$ by letting

$$
\xi=\lambda x-\eta \frac{t^{\mu}}{\mu}
$$

where $\lambda$ and $\eta$ are parameters to be determined later.Now we briefly illustrate the new sub-equation method.

Step 1. Uniting the independent variables $x$ and $t$ into one variable $\xi$ as usual, then from Eq.(3) we obtain

$$
G\left(u, u^{\prime}, u^{\prime \prime}, \ldots\right)=0
$$

Step 2.The solution of Eq. (5) can be expressed by a polynomial in $f(\xi)$ as

$$
u(\xi)=\sum_{j=0}^{\vartheta} b_{j} f^{j}(\xi),
$$


where $b_{j}(0 \leq j \leq \vartheta)$ are constant coefficients to be determined later and $f(\xi)$ satisfies the first order linear ODE of the form

$$
f^{\prime}(\xi)=\alpha+\beta f^{\prime}(\xi)+\sigma f^{\prime \prime}(\xi)
$$

where $\alpha, \beta, \sigma$ are constant. On the other hand, Eq. (7) has the following travelling wave solutions

Family1. If $\beta^{2}-4 \alpha \sigma>0$ and $\sigma \neq 0$, then we have

$$
\begin{aligned}
& f(\xi)=\frac{-\beta}{2 \sigma}-\frac{\sqrt{\beta^{2}-4 \alpha \sigma}}{2 \sigma} \tanh _{p q}\left(\frac{\sqrt{\beta^{2}-4 \alpha \sigma}}{2} \xi\right), \\
& f(\xi)=\frac{-\beta}{2 \sigma}-\frac{\sqrt{\beta^{2}-4 \alpha \sigma}}{2 \sigma} \operatorname{coth}_{p q}\left(\frac{\sqrt{\beta^{2}-4 \alpha \sigma}}{2} \xi\right) .
\end{aligned}
$$

Family 2. If $\beta^{2}-4 \alpha \sigma<0$ and $\sigma \neq 0$, then we have

$$
\begin{aligned}
& f(\xi)=\frac{-\beta}{2 \sigma}+\frac{\sqrt{-\left(\beta^{2}-4 \alpha \sigma\right)}}{2 \sigma} \tan _{p q}\left(\frac{\sqrt{-\left(\beta^{2}-4 \alpha \sigma\right)}}{2} \xi\right), \\
& f(\xi)=\frac{-\beta}{2 \sigma}-\frac{\sqrt{-\left(\beta^{2}-4 \alpha \sigma\right)}}{2 \sigma} \cot _{p q}\left(\frac{\sqrt{-\left(\beta^{2}-4 \alpha \sigma\right)}}{2} \xi\right) .
\end{aligned}
$$

Family 3. If $\alpha \sigma<0, \sigma \neq 0$ and $\beta=0$, then we have

$$
\begin{aligned}
& f(\xi)=-\sqrt{\frac{-\alpha}{\sigma}} \tanh _{p q}(\sqrt{-\alpha \sigma} \xi), \\
& f(\xi)=-\sqrt{\frac{-\alpha}{\sigma}} \operatorname{coth}_{p q}(\sqrt{-\alpha \sigma} \xi) .
\end{aligned}
$$

Family 4. If $\alpha \sigma>0, \sigma \neq 0$ and $\beta=0$, then we have

$$
\begin{aligned}
& f(\xi)=\sqrt{\frac{\alpha}{\sigma}} \tan _{p q}(\sqrt{\alpha \sigma} \xi), \\
& f(\xi)=-\sqrt{\frac{\alpha}{\sigma}} \cot _{p q}(\sqrt{\alpha \sigma} \xi) .
\end{aligned}
$$

Family 5. If $\beta=0$ and $\alpha=-\sigma$, then we have

$$
\begin{gathered}
f(\xi)=-\tanh _{p q}(\alpha \xi), \\
f(\xi)=-\operatorname{coth}_{p q}(\alpha \xi) .
\end{gathered}
$$

Case 6. If $\beta=0$ and $\alpha=\sigma$, then we have

$$
\begin{aligned}
& f(\xi)=\tan _{p q}(\alpha \xi), \\
& f(\xi)=-\cot _{p q}(\alpha \xi) .
\end{aligned}
$$

Family 7. If $\beta^{2}=4 \alpha \sigma$, then we have

$$
f(\xi)=\frac{-2 \alpha(\beta \xi+2)}{\beta^{2} \xi} .
$$

Family8. If $\alpha=0$ and $\beta \neq 0$, then we have

$$
\begin{aligned}
& f(\xi)=-\frac{p \beta}{\sigma\left(\cosh _{p q}(\beta \xi)-\sinh _{p q}(\beta \xi)+p\right)}, \\
& f(\xi)=-\frac{\beta\left(\sinh _{p q}(\beta \xi)+\cosh _{p q}(\beta \xi)\right)}{\sigma\left(\sinh _{p q}(\beta \xi)+\cosh _{p q}(\beta \xi)+q\right)} .
\end{aligned}
$$


Family 9. If $\beta=\alpha=0$, then we have

$$
f(\xi)=\frac{-1}{\sigma \xi} .
$$

Family 10. If $\beta=v, \sigma=m v$ and $\alpha=0$, then we have

$$
f(\xi)=\frac{p e^{v \xi}}{q-p m e^{v \xi}} .
$$

Family11.If $\beta=v, \alpha=m v(m \neq 0)$ and $\sigma=0$, then we have

$$
f(\xi)=e^{v \xi}-m .
$$

Family 12.If $\sigma=0$, then

$$
f(\xi)=e^{\beta \xi}-\frac{\alpha}{\beta} .
$$

Family 13. If $\beta=\sigma=0$, then

$$
f(\xi)=\alpha \xi
$$

The generalized hypergeometric functions and the generalized trigonometric functions are defined as [28]

$$
\begin{aligned}
& \sinh _{A}(\xi)=\frac{p e^{\xi}-q e^{-\xi}}{2}, \cosh _{A}(\xi)=\frac{p e^{\xi}+q e^{-\xi}}{2}, \\
& \tanh _{A}(\xi)=\frac{p e^{\xi}-q e^{-\xi}}{p e^{\xi}+q e^{-\xi}}, \operatorname{coth}_{A}(\xi)=\frac{p e^{\xi}+q e^{-\xi}}{p e^{\xi}-q e^{-\xi}}, \\
& \operatorname{sech}_{A}(\xi)=\frac{2}{p e^{\xi}+q e^{-\xi}}, \operatorname{csch}_{A}(\xi)=\frac{2}{p e^{\xi}-q e^{-\xi}}, \\
& \sin _{A}(\xi)=\frac{p e^{i \xi}-q e^{-i \xi}}{2 i}, \cos _{A}(\xi)=\frac{p e^{i \xi}+q e^{-i \xi}}{2}, \\
& \tan _{A}(\xi)=-i \frac{p e^{i \xi}-q e^{-i \xi}}{p e^{i \xi}+q e^{-i \xi}}, \cot _{A}(\xi)=i \frac{p e^{i \xi}+q e^{-i \xi}}{p e^{i \xi}-q e^{-i \xi}}, \\
& \sec _{A}(\xi)=\frac{2}{p e^{i \xi}+q e^{-i \xi}}, \csc _{A}(\xi)=\frac{2 i}{p e^{i \xi}-q e^{-i \xi}}
\end{aligned}
$$

where $\xi$ is an independent variable, $p, q>0$ are constants. Also, the positive integer $\vartheta$ can be determined by considering the homogeneous balance between the highest order derivative linear term and nonlinear terms appearing in ODE (4).

Step 3. Substituting Eq.(6) into ODE (5), making use of Eq.(7) and setting the coefficients of all powers of $f(\xi)$ to zeros, we will get a system of algebraic equations, from which $\lambda, \eta$ and $b_{j}(0 \leq j \leq \vartheta)$ can be found explicitly.

Step 4. Substituting the values $b_{j}(0 \leq j \leq \vartheta)$ obtained in Step 3 into Eq.(6), we may get all possible solutions.

\section{Exact Solutions for the conformable time-fractional general Kaup-Kupershmidt Equation}

Upon using the transformation

$$
u(x, t)=u(\xi), \quad \xi=\lambda x-\eta \frac{t^{\mu}}{\mu}
$$

Eq. (1) is transferred to

$$
-\eta u^{\prime}+\frac{1}{5} \gamma^{2} \lambda u^{2} u^{\prime}+\frac{5}{2} \gamma \lambda^{3} u^{\prime} u^{\prime \prime}+\gamma \lambda^{3} u u^{\prime \prime \prime}+\lambda^{5} u^{(5)}=0,
$$


where the prime symbolizes the derivation with respect to $\xi$. Considering the homogeneous balance between $u^{2} u^{\prime}$ and $u^{(5)}$ in Eq. (9) we required that $\vartheta+5=3 \vartheta+1$; then $\vartheta=2$; so we can write (6) as

$$
u(\xi)=b_{0}+b_{1} f(\xi)+b_{2} f^{2}(\xi)
$$

Substituting Eq. (10) along with (7) into Eq. (9) and collecting all terms with the same power of $a^{f(\xi)}$ together, the left hand side of Eq. (9) is converted into a polynomial in $f(\xi)$. Equating each coefficient to be zero yields a set of simultaneous algebraic equations. Solving the this algebraic system with respect to the unknowns variables $b_{0}, b_{1}, b_{2}$ and $\eta$ we find the following sets of solutions

Set 1:

$$
b_{0}=-\frac{5}{4} \frac{\lambda^{2}\left(8 \sigma \alpha+\beta^{2}\right)}{\gamma}, \quad b_{1}=-15 \frac{\lambda^{2} \beta \sigma}{\gamma}, \quad b_{2}=-15 \frac{\lambda^{2} \sigma^{2}}{\gamma}, \quad \eta=\frac{\lambda^{5}}{16}\left(\beta^{2}-4 \sigma \alpha\right)^{2} .
$$

Set 2:

$$
b_{0}=-\frac{10 \lambda^{2}\left(8 \sigma \alpha+\beta^{2}\right)}{\gamma}, \quad b_{1}=-120 \frac{\lambda^{2} \beta \sigma}{\gamma}, \quad b_{2}=-120 \frac{\lambda^{2} \sigma^{2}}{\gamma}, \quad \eta=11 \lambda^{5}\left(\beta^{2}-4 \sigma \alpha\right)^{2} .
$$

Substituting Set 1 along with (8) into (10) and solutions of ODE (7), we have following travelling wave solutions of Eq. (1).

Case1. When $\beta^{2}-4 \alpha \sigma>0$ and $\sigma \neq 0$, then

$$
\begin{aligned}
& u(x, t)=\frac{5 \lambda^{2} \Delta}{4 \gamma}\left(2-3 \tanh _{p q}^{2}\left(\frac{\sqrt{\Delta}}{2}\left(\lambda x-\frac{\lambda^{5}}{16} \Delta^{2} \frac{t^{\mu}}{\mu}\right)\right)\right), \\
& u(x, t)=\frac{5 \lambda^{2} \Delta}{4 \gamma}\left(2-3 \operatorname{coth}_{p q}^{2}\left(\frac{\sqrt{\Delta}}{2}\left(\lambda x-\frac{\lambda^{5}}{16} \Delta^{2} \frac{t^{\mu}}{\mu}\right)\right)\right),
\end{aligned}
$$

where $\Delta=\beta^{2}-4 \alpha \sigma$.

Case 2. When $\beta^{2}-4 \alpha \sigma<0$ and $\sigma \neq 0$, then

$$
\begin{aligned}
& u(x, t)=\frac{5 \lambda^{2} \Delta}{4 \gamma}\left(2+3 \tan _{p q}^{2}\left(\frac{\sqrt{-\Delta}}{2}\left(\lambda x-\frac{\lambda^{5}}{16} \Delta^{2} \frac{t^{\mu}}{\mu}\right)\right)\right), \\
& u(x, t)=\frac{5 \lambda^{2} \Delta}{4 \gamma}\left(2-3 \cot _{p q}^{2}\left(\frac{\sqrt{-\Delta}}{2}\left(\lambda x-\frac{\lambda^{5}}{16} \Delta^{2} \frac{t^{\mu}}{\mu}\right)\right)\right),
\end{aligned}
$$

where $\Delta=\beta^{2}-4 \alpha \sigma$.

Case 3. When $\alpha \sigma<0, \sigma \neq 0$ and $\beta=0$, then

$$
\begin{aligned}
& u(x, t)=\frac{5 \lambda^{2} \sigma \alpha}{\gamma}\left(-2+3 \tanh _{p q}^{2}\left(\sqrt{-\sigma \alpha}\left(\lambda x-\lambda^{5}(\sigma \alpha)^{2} \frac{t^{\mu}}{\mu}\right)\right)\right), \\
& u(x, t)=\frac{5 \lambda^{2} \sigma \alpha}{\gamma}\left(-2+3 \operatorname{coth}_{p q}^{2}\left(\sqrt{-\sigma \alpha}\left(\lambda x-\lambda^{5}(\sigma \alpha)^{2} \frac{t^{\mu}}{\mu}\right)\right)\right) .
\end{aligned}
$$

Case 4. When $\alpha \sigma>0, \sigma \neq 0$ and $\beta=0$, then

$$
\begin{aligned}
& u(x, t)=-\frac{5 \lambda^{2} \sigma \alpha}{\gamma}\left(2+3 \tan _{p q}^{2}\left(\sqrt{\sigma \alpha}\left(\lambda x-\lambda^{5}(\sigma \alpha)^{2} \frac{t^{\mu}}{\mu}\right)\right)\right), \\
& u(x, t)=\frac{5 \lambda^{2} \sigma \alpha}{\gamma}\left(-2+3 \cot _{p q}^{2}\left(\sqrt{\sigma \alpha}\left(\lambda x-\lambda^{5}(\sigma \alpha)^{2} \frac{t^{\mu}}{\mu}\right)\right)\right) .
\end{aligned}
$$


Case 5. When $\beta=0$ and $\alpha=-\sigma$, then

$$
\begin{aligned}
& u(x, t)=-\frac{5 \lambda^{2} \alpha^{2}}{\gamma}\left(-2+3 \tanh _{p q}^{2}\left(\alpha\left(\lambda x-\lambda^{5} \alpha^{4} \frac{t^{\mu}}{\mu}\right)\right)\right), \\
& u(x, t)=-\frac{5 \lambda^{2} \alpha^{2}}{\gamma}\left(-2+3 \operatorname{coth}_{p q}^{2}\left(\alpha\left(\lambda x-\lambda^{5} \alpha^{4} \frac{t^{\mu}}{\mu}\right)\right)\right),
\end{aligned}
$$

Case 6. When $\beta=0$ and $\alpha=\sigma$, then

$$
\begin{aligned}
& u(x, t)=-\frac{5 \lambda^{2} \alpha^{2}}{\gamma}\left(2+3 \tan _{p q}^{2}\left(\alpha\left(\lambda x-\lambda^{5} \alpha^{4} \frac{t^{\mu}}{\mu}\right)\right)\right), \\
& u(x, t)=-\frac{5 \lambda^{2} \alpha^{2}}{\gamma}\left(-2+3 \cot _{p q}^{2}\left(\alpha\left(\lambda x-\lambda^{5} \alpha^{4} \frac{t^{\mu}}{\mu}\right)\right)\right),
\end{aligned}
$$

Case 8. When $\alpha=0$ and $\beta \neq 0$, then

$$
\begin{aligned}
u(x, t)= & \frac{10 \lambda^{2} \beta^{2}}{\gamma}\left(-\frac{1}{4}+\frac{12 p}{\left(\cosh _{p q}\left(\beta\left(\lambda x-\frac{\lambda^{5} \beta^{4}}{16} \frac{t^{\mu}}{\mu}\right)\right)-\sinh _{p q}\left(\beta\left(\lambda x-\frac{\lambda^{5} \beta^{4}}{16} \frac{t^{\mu}}{\mu}\right)\right)+p\right)}\right. \\
& \left.-12\left(-\frac{p}{\left(\cosh _{p q}\left(\beta\left(\lambda x-\frac{\lambda^{5} \beta^{4}}{16} \frac{t^{\mu}}{\mu}\right)\right)-\sinh _{p q}\left(\beta\left(\lambda x-\frac{\lambda^{5} \beta^{4}}{16} \frac{t^{\mu}}{\mu}\right)\right)+p\right)}\right)^{2}\right), \\
u(x, t)= & \frac{10 \lambda^{2} \beta^{2}}{\gamma}\left(-\frac{1}{4}+\frac{12\left(\sinh _{p q}\left(\beta\left(\lambda x-\frac{\lambda^{5} \beta^{4}}{16} \frac{t^{\mu}}{\mu}\right)\right)+\cosh _{p q}\left(\beta\left(\lambda x-\frac{\lambda^{5} \beta^{4}}{16} \frac{t^{\mu}}{\mu}\right)\right)\right)}{\left(\sinh _{p q}\left(\beta\left(\lambda x-\frac{\lambda^{5} \beta^{4}}{16} \frac{t^{\mu}}{\mu}\right)\right)+\cosh _{p q}\left(\beta\left(\lambda x-\frac{\lambda^{5} \beta^{4}}{16} \frac{t^{\mu}}{\mu}\right)\right)+q\right)}\right. \\
& -12\left(\frac{\left(\sinh _{p q}\left(\beta\left(\lambda x-\frac{\lambda^{5} \beta^{4}}{16} \frac{t^{\mu}}{\mu}\right)\right)+\cosh _{p q}\left(\beta\left(\lambda x-\frac{\lambda^{5} \beta^{4}}{16} \frac{t^{\mu}}{\mu}\right)\right)\right)}{\left(\sinh _{p q}\left(\beta\left(\lambda x-\frac{\lambda^{5} \beta^{4}}{16} \frac{t^{\mu}}{\mu}\right)\right)+\cosh _{p q}\left(\beta\left(\lambda x-\frac{\lambda^{5} \beta^{4}}{16} \frac{t \mu}{\mu}\right)\right)+q\right)}\right) .
\end{aligned}
$$

Case 10. When beta $=v, \sigma=m v$ and $\alpha=0$ then

$$
u(x, t)=-\frac{10 \lambda^{2} v^{2}}{\gamma}\left(\frac{1}{4}+12 m \frac{p e^{v\left(\lambda x-\frac{\lambda^{5} v^{4}}{16} \frac{t^{\mu}}{\mu}\right)}}{q-p m e^{v\left(\lambda x-\frac{\lambda^{5} v^{4}}{16} \frac{t^{\mu}}{\mu}\right)}}+12 m\left(\frac{p e^{v\left(\lambda x-\frac{\lambda^{5} v^{4}}{16} \frac{t^{\mu}}{\mu}\right)}}{q-p m e^{v\left(\lambda x-\frac{\lambda^{5} v^{4}}{16} \frac{t^{\mu}}{\mu}\right)}}\right)^{2}\right) .
$$

Substituting Set 2 along with (8) into (10) and solutions of ODE (7), we have following travelling wave solutions of Eq. (1).

Case1. When $\beta^{2}-4 \alpha \sigma>0$ and $\sigma \neq 0$, then

$$
\begin{aligned}
& u(x, t)=\frac{10 \lambda^{2} \Delta}{\gamma}\left(2-3 \tanh _{p q}^{2}\left(\frac{\sqrt{\Delta}}{2}\left(\lambda x-11 \lambda^{5} \Delta^{2} \frac{t^{\mu}}{\mu}\right)\right)\right), \\
& u(x, t)=\frac{10 \lambda^{2} \Delta}{\gamma}\left(2-3 \operatorname{coth}_{p q}^{2}\left(\frac{\sqrt{\Delta}}{2}\left(\lambda x-11 \lambda^{5} \Delta^{2} \frac{t^{\mu}}{\mu}\right)\right)\right),
\end{aligned}
$$

where $\Delta=\beta^{2}-4 \alpha \sigma$.

Case 2. When $\beta^{2}-4 \alpha \sigma<0$ and $\sigma \neq 0$, then

$$
\begin{aligned}
& u(x, t)=\frac{10 \lambda^{2} \Delta}{\gamma}\left(2+3 \tan _{p q}^{2}\left(\frac{\sqrt{-\Delta}}{2}\left(\lambda x-11 \lambda^{5} \Delta^{2} \frac{t^{\mu}}{\mu}\right)\right)\right), \\
& u(x, t)=\frac{10 \lambda^{2} \Delta}{\gamma}\left(2-3 \cot _{p q}^{2}\left(\frac{\sqrt{-\Delta}}{2}\left(\lambda x-11 \lambda^{5} \Delta^{2} \frac{t^{\mu}}{\mu}\right)\right)\right),
\end{aligned}
$$

where $\Delta=\beta^{2}-4 \alpha \sigma$.

Case 3. When $\alpha \sigma<0, \sigma \neq 0$ and $\beta=0$, then

$$
u(x, t)=\frac{40 \lambda^{2} \sigma \alpha}{\gamma}\left(-2+3 \tanh _{p q}^{2}\left(\sqrt{-\sigma \alpha}\left(\lambda x-176 \lambda^{5}(\sigma \alpha)^{2} \frac{t^{\mu}}{\mu}\right)\right)\right),
$$




$$
u(x, t)=\frac{40 \lambda^{2} \sigma \alpha}{\gamma}\left(-2+3 \operatorname{coth}_{p q}^{2}\left(\sqrt{-\sigma \alpha}\left(\lambda x-176 \lambda^{5}(\sigma \alpha)^{2} \frac{t^{\mu}}{\mu}\right)\right)\right)
$$

Case 4. When $\alpha \sigma>0, \sigma \neq 0$ and $\beta=0$, then

$$
\begin{aligned}
& u(x, t)=-\frac{40 \lambda^{2} \sigma \alpha}{\gamma}\left(2+3 \tan _{p q}^{2}\left(\sqrt{\sigma \alpha}\left(\lambda x-176 \lambda^{5}(\sigma \alpha)^{2} \frac{t^{\mu}}{\mu}\right)\right)\right) \\
& u(x, t)=\frac{40 \lambda^{2} \sigma \alpha}{\gamma}\left(-2+3 \cot _{p q}^{2}\left(\sqrt{\sigma \alpha}\left(\lambda x-176 \lambda^{5}(\sigma \alpha)^{2} \frac{t^{\mu}}{\mu}\right)\right)\right)
\end{aligned}
$$

Case 5. When $\beta=0$ and $\alpha=-\sigma$, then

$$
\begin{aligned}
& u(x, t)=-\frac{40 \lambda^{2} \alpha^{2}}{\gamma}\left(-2+3 \tanh _{p q}^{2}\left(\alpha\left(\lambda x-176 \lambda^{5} \alpha^{4} \frac{t^{\mu}}{\mu}\right)\right)\right), \\
& u(x, t)=-\frac{40 \lambda^{2} \alpha^{2}}{\gamma}\left(-2+3 \operatorname{coth}_{p q}^{2}\left(\alpha\left(\lambda x-176 \lambda^{5} \alpha^{4} \frac{t^{\mu}}{\mu}\right)\right)\right),
\end{aligned}
$$

Case 6. When $\beta=0$ and $\alpha=\sigma$, then

$$
\begin{gathered}
u(x, t)=-\frac{40 \lambda^{2} \alpha^{2}}{\gamma}\left(2+3 \tan _{p q}^{2}\left(\alpha\left(\lambda x-176 \lambda^{5} \alpha^{4} \frac{t^{\mu}}{\mu}\right)\right)\right), \\
u(x, t)=-\frac{40 \lambda^{2} \alpha^{2}}{\gamma}\left(-2+3 \cot _{p q}^{2}\left(\alpha\left(\lambda x-176 \lambda^{5} \alpha^{4} \frac{t^{\mu}}{\mu}\right)\right)\right),
\end{gathered}
$$

Case 8. When $\alpha=0$ and $\beta \neq 0$, then

$$
\begin{aligned}
u(x, t)= & \frac{10 \lambda^{2} \beta^{2}}{\gamma}\left(-\frac{1}{4}+\frac{12 p}{\left(\cosh _{p q}\left(\beta\left(\lambda x-11 \lambda^{5} \beta^{4} \frac{t^{\mu}}{\mu}\right)\right)-\sinh _{p q}\left(\beta\left(\lambda x-11 \lambda^{5} \beta^{4} \frac{t^{\mu}}{\mu}\right)\right)+p\right)}\right. \\
& \left.-12\left(-\frac{p}{\left(\cosh _{p q}\left(\beta\left(\lambda x-11 \lambda^{5} \beta^{4} \frac{t^{\mu}}{\mu}\right)\right)-\sinh _{p q}\left(\beta\left(\lambda x-11 \lambda^{5} \beta^{4} \frac{t^{\mu}}{\mu}\right)\right)+p\right)}\right)^{2}\right), \\
u(x, t)= & \frac{10 \lambda^{2} \beta^{2}}{\gamma}\left(-\frac{1}{4}+\frac{12\left(\sinh _{p q}\left(\beta\left(\lambda x-11 \lambda^{5} \beta^{4} \frac{t^{\mu}}{\mu}\right)\right)+\cosh _{p q}\left(\beta\left(\lambda x-11 \lambda^{5} \beta^{4} \frac{t^{\mu}}{\mu}\right)\right)\right)}{\left(\sinh _{p q}\left(\beta\left(\lambda x-11 \lambda^{5} \beta^{4} \frac{t^{\mu}}{\mu}\right)\right)+\cosh _{p q}\left(\beta\left(\lambda x-11 \lambda^{5} \beta^{4} \frac{t^{\mu}}{\mu}\right)\right)+q\right)}\right. \\
& \left.-12\left(\frac{\left(\sinh _{p q}\left(\beta\left(\lambda x-11 \lambda^{5} \beta^{4} \frac{t^{\mu}}{\mu}\right)\right)+\cosh _{p q}\left(\beta\left(\lambda x-11 \lambda^{5} \beta^{4} \frac{t^{\mu}}{\mu}\right)\right)\right)}{\left(\sinh _{p q}\left(\beta\left(\lambda x-11 \lambda^{5} \beta^{4} \frac{t^{\mu}}{\mu}\right)\right)+\cosh _{p q}\left(\beta\left(\lambda x-11 \lambda^{5} \beta^{4} \frac{t^{\mu}}{\mu}\right)\right)+q\right)}\right)^{2}\right) .
\end{aligned}
$$

Case 10. When $\beta=v, \sigma=m v$ and $\alpha=0$ then

$$
u(x, t)=-\frac{10 \lambda^{2} v^{2}}{\gamma}\left(\frac{1}{4}+12 m \frac{p e^{v\left(\lambda x-11 \lambda^{5} v^{4} \frac{t^{\mu}}{\mu}\right)}}{q-p m e^{v\left(\lambda x-11 \lambda^{5} v^{4} \frac{t^{\mu}}{\mu}\right)}}+12 m\left(\frac{p e^{v\left(\lambda x-11 \lambda^{5} v^{4} \frac{t^{\mu}}{\mu}\right)}}{q-p m e^{v\left(\lambda x-11 \lambda^{5} v^{4} \frac{t^{\mu}}{\mu}\right)}}\right)^{2}\right) .
$$

\section{Conclusions}

The new sub-equation method has been successfully employed to time fractional general Kaup-Kuperschmidt equation. Authors fistly used chain rule and wave transform so the nonlinear conformable fractional differential equation turns into differential equation with integer order derivative. All the results show that both arguments(chain rule, wave transform, new sub-equation method) are applicable, reliable are efficient tools for obtaining the exact solutions of nonlinear partial differential equations with conformable fractional derivative. 


\section{REFERENCES}

[1] K. Oldham and J. Spanier, The Fractional Calculus, Theory and Applications of Differentiation and Integration of Arbitrary Order, Academic Press, 1974

[2] K.S. Miller, B. Ross, An Introduction to the Fractional Calculus and Fractional Differential Equations, A Wiley-Interscience Publication, 1993

[3] I. Podlubny, Fractional Differential Equations, Academic Press, 1999

[4] A.A. Kilbas, H.M. Srivastava and J.J. Trujillo, Theory and Applications of Fractional Differential Equations, Elsevier, 2006.

[5] Kurt, A., Tasbozan, O., Approximate Analytical Solution of the Time Fractional Whitham-Broer-Kaup Equation Using the Homotopy Analysis Method, Int. J. Pure Appl. Math. 98 (2015), 503-510.

[6] Çelik, C., Duman, M., Crank-Nicolson method for the fractional diffusion equation with the Riesz fractional derivative, J. Comput. Phys. 231 (2012), 1743-1750.

[7] Tasbozan, O., Esen, A., Yagmurlu, N. M., Ucar, Y., A numerical solution to fractional diffusion equation for force-free case, Abstr. Appl. Anal. 2013 (2013).

[8] Cenesiz, Y., Kurt, A., New fractional complex transform for conformable fractional partial differential equations, J. Appl. Math. Stat. Inf. $12(2016), 41-47$.

[9] Abdeljawad, T., On conformable fractional calculus, J. Comput. Appl. Math. 279 (2015), 57-66.

[10] Korkmaz, A., Hosseini, K., Exact solutions of a nonlinear conformable time-fractional parabolic equation with exponential nonlinearity using reliable methods, Optical Quantum Electron. 49 (2017, 278

[11] Cenesiz, Y., Baleanu, D., Kurt, A., Tasbozan, O., New exact solutions of Burgers' type equations with conformable derivative, Waves in Random and Complex Media, 27 (2017), 103-116.

[12] Kurt, A., Tasbozan, O., Baleanu, D., New solutions for conformable fractional Nizhnik-Novikov-Veselov system via $G^{\prime} / G$ expansion method and homotopy analysis methods, Optical Quantum Electron. 49 (2017), 333.

[13] Hosseini, K., Mayeli, P., Ansari, R., Bright and singular soliton solutions of the conformable time-fractional Klein-Gordon equations with different nonlinearities, Waves in Random and Complex Media, 28 (2018), 426-434.

[14] Rezazadeh, H., Manafian, J., Khodadad, F. S., Nazari, F., Traveling wave solutions for density-dependent conformable fractional diffusion-reaction equation by the first integral method and the improved $\tan \left(\frac{1}{2} \varphi(\xi)\right)$-expansion method, Optical Quantum Electron. 50 (2018), 121.

[15] Osman, M. S., Korkmaz, A., Rezazadeh, H., Mirzazadeh, M., Eslami, M., Zhou, Q., The Unified Method for Conformable Time Fractional Schrdinger Equation with Perturbation Terms, Chinese J. Phys. 56 (2018), 2500-2506.

[16] Rezazadeh, H., Tariq, H., Eslami, M., Mirzazadeh, M., Zhou, Q., New exact solutions of nonlinear conformable time-fractional Phi-4 equation, Chinese J. Phys. 56 (2018), 2805-2816

[17] Bulut, H., Sulaiman, T. A., Baskonus, H. M., Rezazadeh, H., Eslami, M., Mirzazadeh, M., Optical solitons and other solutions to the conformable space-time fractional Fokas-Lenells equation. Optik, 172 (2018), 20-27.

[18] Rezazadeh, H., Mirhosseini-Alizamini, S. M., Eslami, M., Rezazadeh, M., Mirzazadeh, M., Abbagari, S., New optical solitons of nonlinear conformable fractional Schrödinger-Hirota equation, Optik, 172 (2018), 545-553.

[19] Aminikhah, H., Sheikhani, A. R., Rezazadeh, H., Sub-equation method for the fractional regularized long-wave equations with conformable fractional derivatives, Scientia Iranica. Trans. B, Mech. Eng. 23 (2016), 1048.

[20] Rezazadeh, H., Osman, M. S., Eslami, M., Ekici, M., Sonmezoglu, A., Asma, M., Othman, W.A.M., Wong, B.R., Mirzazadeh M.,Zhou, Q., Biswas, A., Belic, M., Mitigating Internet bottleneck with fractional temporal evolution of optical solitons having quadratic-cubic nonlinearity, Optik, 164 (2018), 84-92.

[21] Biswas, A., Al-Amr, M. O., Rezazadeh, H., Mirzazadeh, M., Eslami, M., Zhou, Q., Moshokoa, S. P., Belic, M. Resonant optical solitons with dual-power law nonlinearity and fractional temporal evolution, Optik 165 (2018), 233-239.

[22] Bulut, H., Sulaiman, T. A., Baskonus, H. M., Dark, bright optical and other solitons with conformable space-time fractional second-order spatiotemporal dispersion, Optik, 163 (2018), 1-7. 
[23] Wazwaz, A. M., The extended tanh method for new solitons solutions for many forms of the fifth-order KdV equations, Appl. Math. Comput. 184 (2007), 1002-1014.

[24] Feng, D., Li, K., On Exact Traveling Wave Solutions for (1 + 1) Dimensional Kaup-Kupershmidt Equation, Appl. Math. 2 (2011), $752-756$.

[25] Gomez S, C. A., New traveling waves solutions to generalized Kaup-Kupershmidt and Ito equations, Appl. Math. Comput. 216 (2010), 241-250.

[26] Salas, A. H., Solving the Generalized Kaup-Kupershmidt Equation, Adv. Studies Theor. Phys, 6 (2012), 879-885.

[27] Khalil, R., Al Horani, M., Yousef, A., Sababheh, M., A new definition of fractional derivative, J. Comput. Appl. Math. 264 (2014), 65-70.

[28] Rezazadeh, H., Korkmaz, A., Eslami, M., Vahidi, J., Asghari, R., Traveling wave solution of conformable fractional generalized reaction Duffing model by generalized projective Riccati equation method, Optical Quantum Electron. 50 (2018), 150. 